\title{
Experimental comparison of piezoelectric and magnetostrictive shunt dampers
}

Vivake M. Asnani

NASA Glenn Research Center

Materials and Structures Division, Rotating and Drive Systems Branch

Cleveland, OH 44135 USA, vivake.m.asnani@nasa.gov

\section{Zhangxian Deng and Marcelo J. Dapino}

NSF Industry \& University Cooperative Research Center on Smart Vehicle Concepts

The Ohio State University, Department of Mechanical \& Aerospace Engineering

Columbus, OH 43210 USA

\section{Justin J. Scheidler}

Universities Space Research Association at NASA Glenn Research Center

Materials and Structures Division, Rotating and Drive Systems Branch

Cleveland, $\mathrm{OH} 44135$ USA 


\section{Outline}

- Introduction

$>$ Motivation, objectives, and scope

- Experiment

$>$ Load frame testing of shunt dampers

- Results

$>$ Frequency response comparison

- Summary and conclusions 


\section{Outline}

- Introduction

$>$ Motivation, objectives, and scope

- Experiment

$>$ Load frame testing of shunt dampers

- Results

$>$ Frequency response comparison

- Summary and conclusions 


\section{Driveline vibration effects}

- Vibration is a side effect of transferring power through a rotating driveline.

- It causes functional issues, like reduced precision in cutting tools.

- Vibration generated by rotorcraft gearing causes cabin noise in excess of $100 \mathrm{~dB}$ !

- This environment prohibits widespread use of rotorcraft for civilian transportation.
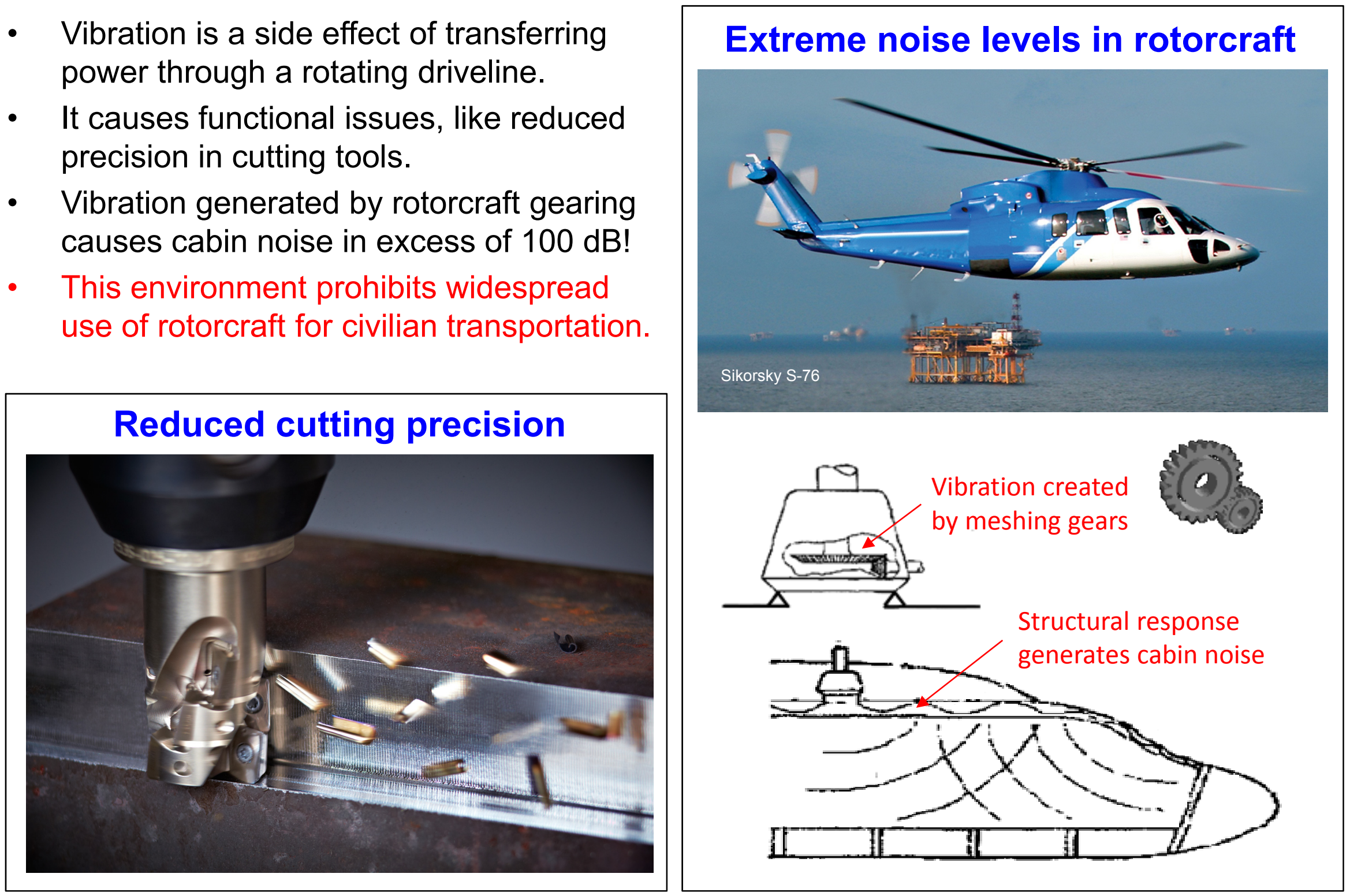

National Aeronautics and Space Administration 


\section{Driveline damping using the vibration ring}

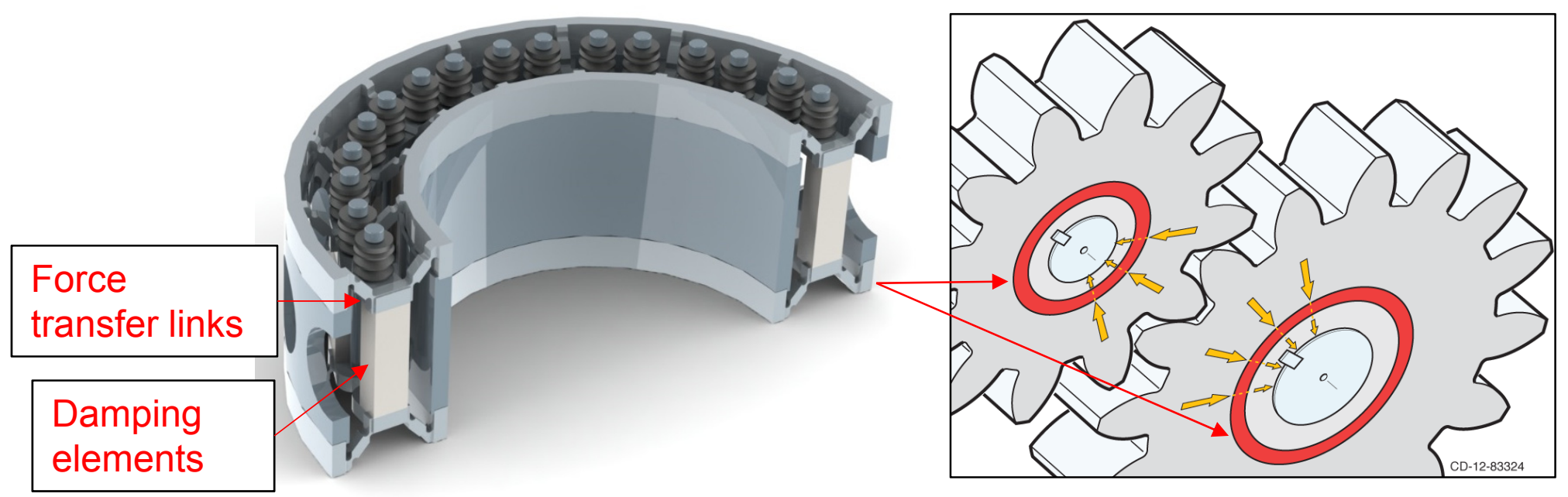

- The vibration ring is designed to incorporate damping elements into a driveline

- Force is transferred through the elements to create vibration isolation and damping

- Damping elements must have high stiffness to maintain the driveline alignment.

Material property comparison

\begin{tabular}{|rlll|}
\hline Application & Material & Modulus (GPa) & Loss factor \\
\hline Driveline components & Steel & 200 & 0.0005 \\
\hline Vibration damping treatment & Rubber & 0.05 & 0.50 \\
\hline Vibration ring damping elements & TBD & 5 to 35 & Maximize \\
\hline
\end{tabular}




\section{Shunt damper options}

- High stiffness smart materials: Piezoelectric ceramics and magnetostrictive metals

- Electrical $\Leftrightarrow$ mechanical, Magnetic $\Leftrightarrow$ mechanical

Piezoelectric schematic

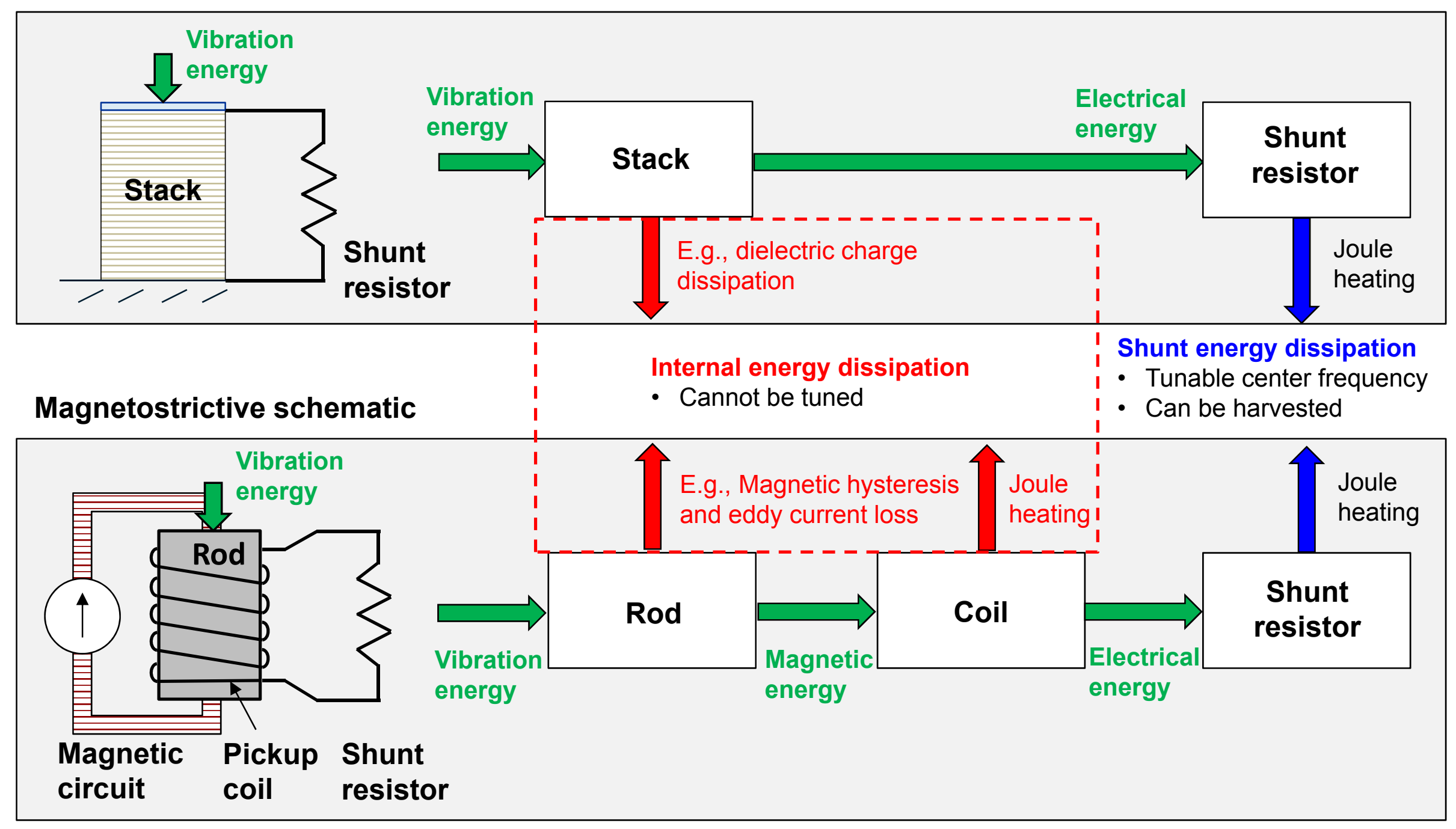

National Aeronautics and Space Administration

\section{Energy flow diagrams}




\section{Objectives and scope}

- Objective : Characterize 3 candidate shunt damping devices

- Maximize damping at $750 \mathrm{~Hz}$

- Measure electro-mechanical response to vibratory force up $1000 \mathrm{~Hz}$

$>$ Stiffness, damping

$>$ Internal vs. shunt energy dissipation 


\section{Outline}

- Introduction

$>$ Motivation, objectives, and scope

- Experiment

$>$ Load frame testing of shunt dampers

- Results

$>$ Frequency response comparison

- Summary and conclusions 


\section{Test articles}

\section{Piezoelectric shunt dampers}

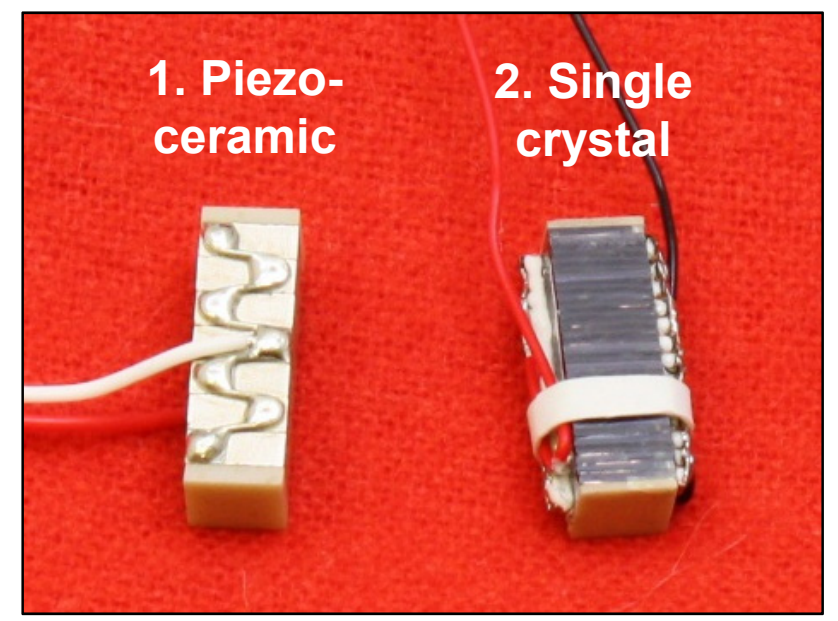

1. Piezoceramic: Soft-doped polycrystalline co-fired lead zirconate titanate (PZT)

2. Single crystal: Lead magnesium niobate-lead titanate (PMN-30\%PT)

\section{Magnetostrictive shunt damper}

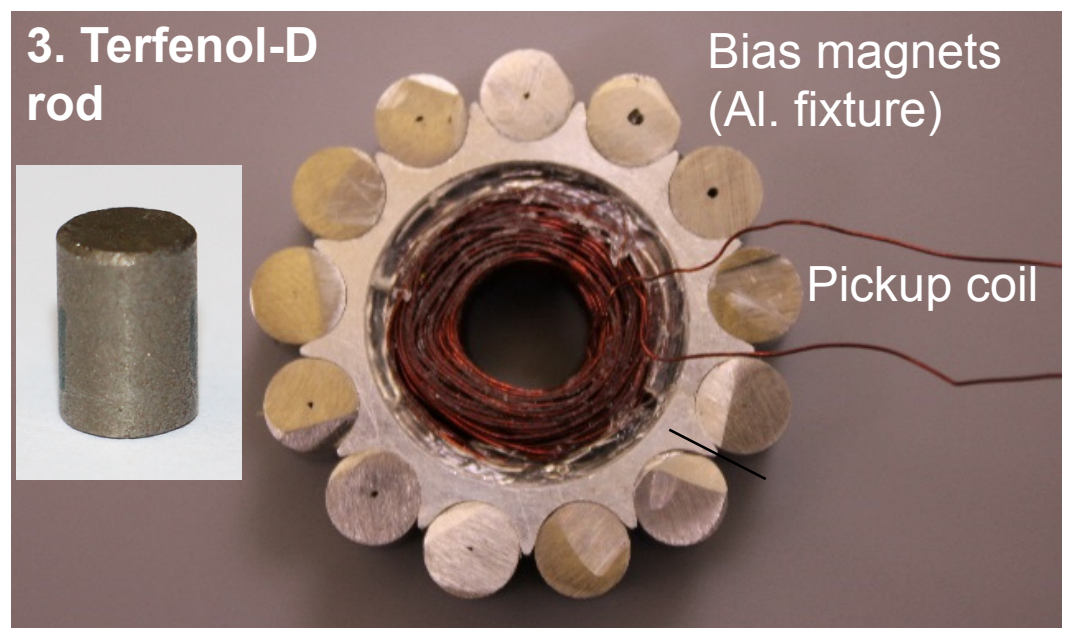

3. Terfenol-D

- Terbium, dysprosium and iron rod $\left(\mathrm{Tb}_{0.3} \mathrm{Dy}_{0.7} \mathrm{Fe}_{1.92}\right)$

- Alnico grade 8 magnets

- Optimized (500-turn 30AWG) pickup coil

- Nominal: $7 \mathrm{~mm}$ diameter, $10 \mathrm{~mm}$ long

- Nominal: $5 \mathrm{~mm} \times 5 \mathrm{~mm} \times 16 \mathrm{~mm}$ 


\section{Test setup}

Dynamic load frame assembly

-Piezoceramic case-

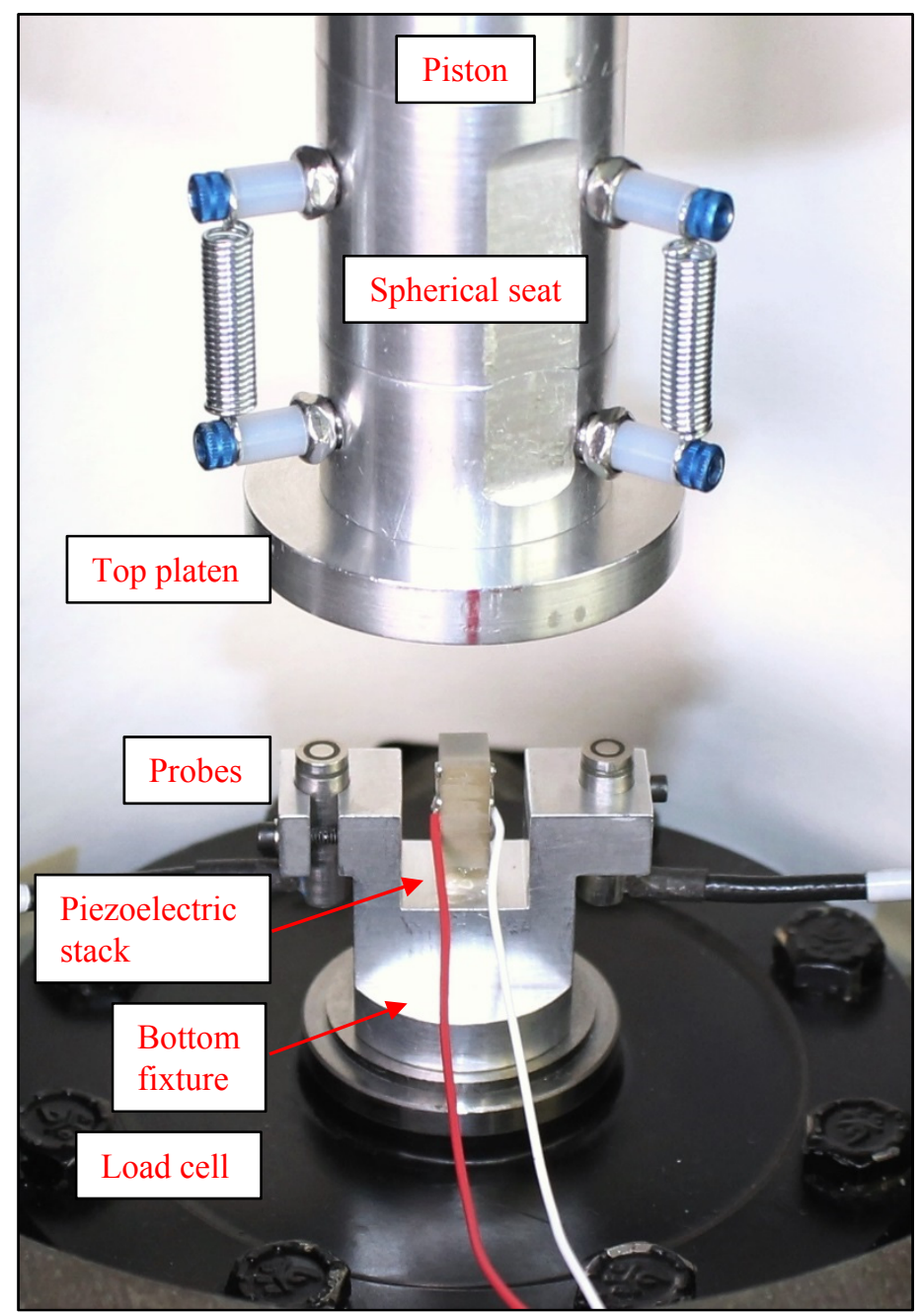

Provision to minimize error

- Even pressure on sample face

- Minimized inertial force error

- Magneto setup: Moving magnets

$>$ Attractive forces did not corrupt force

$>$ Did not generate voltage error

- Sensor channels were phase aligned

Removed data influenced by resonance

- Resonance at 1.0 to $1.2 \mathrm{kHz}$

- Maximum data

> Piezoceramic $923 \mathrm{~Hz}$

$>$ Single crystal $804 \mathrm{~Hz}$

> Terfenol-D $350 \mathrm{~Hz}$ (higher harmonics) 


\section{Data processing}

$\begin{aligned} & \text { Effective } \\ & \text { compressive modulus }\end{aligned}=\left(\frac{\text { height }}{\text { area }}\right)$ stiffness

$\begin{aligned} & \text { Total } \\ & \text { loss factor }\end{aligned}=\frac{\text { Total energy dissipated } / 2 \pi}{\text { Oscillation energy }}\left\{\begin{array}{l}\begin{array}{l}\text { Internal } \\ \text { loss factor }\end{array}=\frac{\text { Internal energy dissipated } / 2 \pi}{\text { Oscillation energy }} \\ \begin{array}{l}\text { Shunt } \\ \text { loss factor }\end{array}=\frac{\text { Shunt energy dissipated } / 2 \pi}{\text { Oscillation energy }}\end{array}\right.$

- Both contribute to damping

- High shunt loss factor required for tuning damping frequency or for energy harvesting 


\section{Test stages}

\section{Optimize prestress}

- Maximize energy conversion

2. Optimizing resistance at $750 \mathrm{~Hz}$

- Maximize shunt loss factor

3. Measuring frequency response

- Optimal prestress \& optimal shunt resistance

- Frequency varied in steps from $2 \mathrm{~Hz}$ to $1000 \mathrm{~Hz}$

Refer to manuscript

for details

- Compute metrics

Nominal dynamic stress amplitude

Piezoceramic: $8.0 \mathrm{MPa}$

Single crystal: $4.0 \mathrm{MPa}$

Terfenol-D: $\quad 7.3 \mathrm{MPa}$ 


\section{Outline}

- Introduction

$>$ Motivation, objectives, and scope

- Experiment

$>$ Load frame testing of shunt dampers

- Results

$>$ Frequency response comparison

- Summary and conclusions 


\section{Frequency response (1 of 2)}

\section{Modulus}

- Quasi-static: Piezoceramic roughly 2x Single Crystal and Terfenol-D

- Piezoceramic and Single Crystal trends: Increase with frequency. Expected based on electric-charge stiffening

- Terfenol-D trend: Decreases and then increases after $100 \mathrm{~Hz}$. Increase is explained by magnetic field stiffening. Initial decrease is unexplained.

\section{Internal loss factor}

- Quasi-static: Terfenol-D > Single crystal > Piezoceramic

- Piezoceramic and Single Crystal trends: Slight inverse relationship with modulus.

- Terfenol-D trend: Unexpected, sharp increase after $30 \mathrm{~Hz}$. 3D COMSOL simulation indicates magnetic energy inducing eddy currents in aluminum magnet fixture

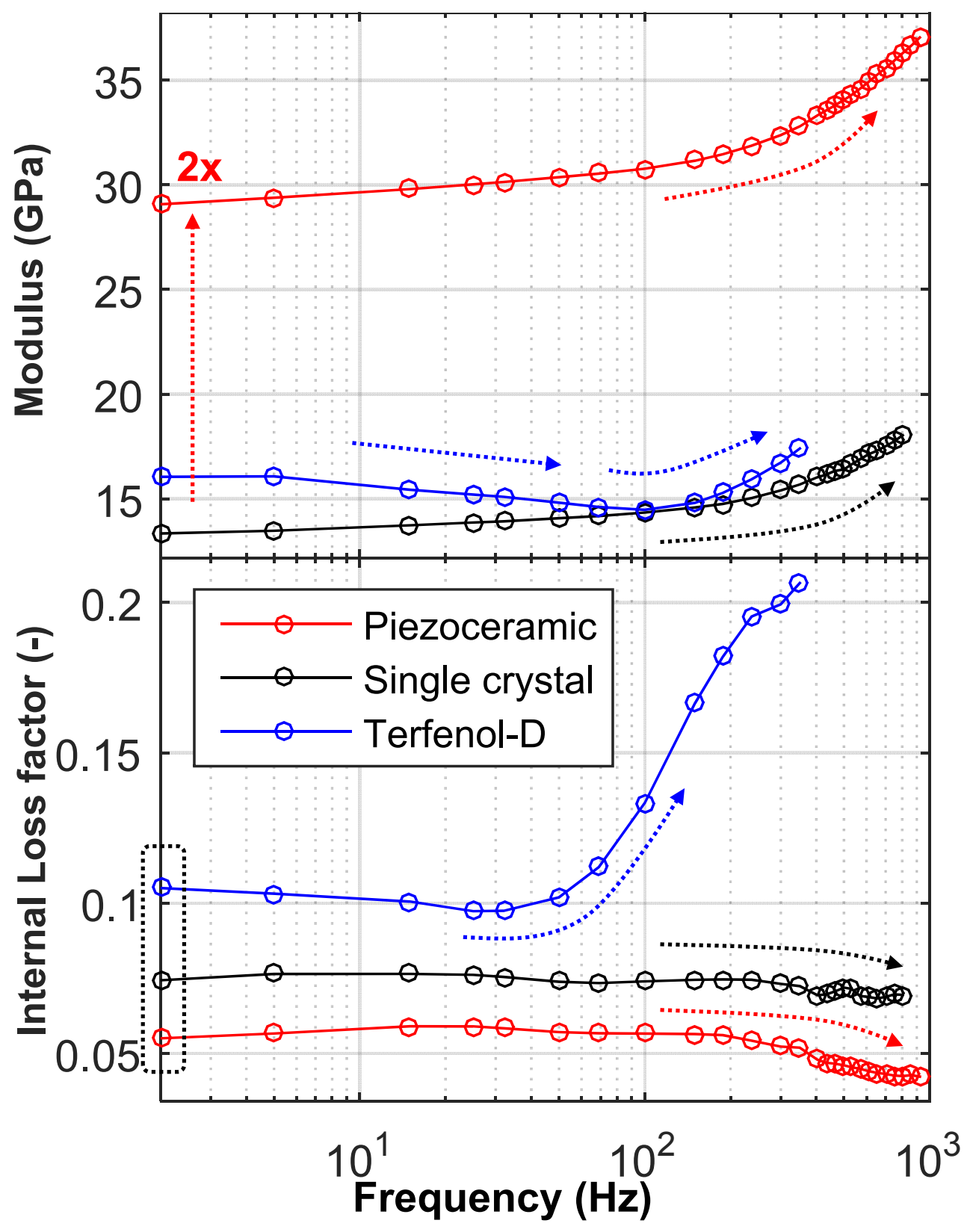




\section{Frequency response (2 of 2)}

\section{Shunt loss factor}

- Peak: Near $750 \mathrm{~Hz}$

Single crystal $>$ Piezoceramic $>$ Terfenol-D

- Piezoceramic and single crystal:

Peak shunt losses >> internal losses

Potential for energy harvesting

- Terfenol-D

Relatively low shunt loss.

Result of eddy current dissipation

\section{$\underline{\text { Total loss factor }}$}

- All devices: Same order of magnitude as rubber.

\section{- Terfenol-D}

$>$ Highest total loss across all frequencies

$>$ Dominated by eddy current losses

- Peak not tunable

- Coil and shunt not needed

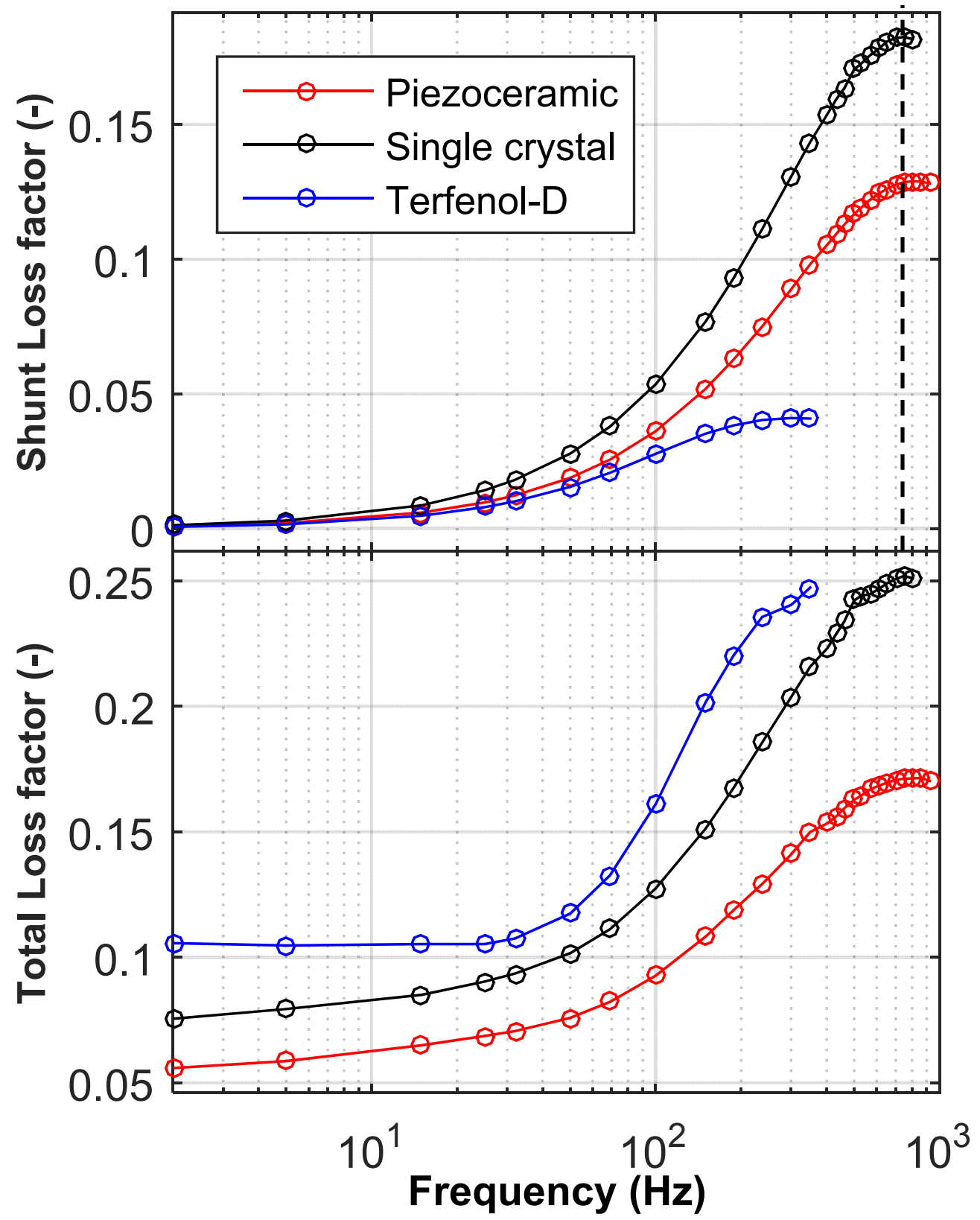




\section{Outline}

- Introduction

$>$ Motivation, objectives, and scope

- Experiment

$>$ Load frame testing of shunt dampers

- Results

$>$ Frequency response comparison

- Summary and conclusions 


\section{Summary}

- Evaluated three high-stiffness shunt damping devices.

Piezoelectric stacks

$>$ Piezoceramic (PZT)

$>$ Single crystal $(\mathrm{PMN}-30 \% \mathrm{PT})$

Magnetostrictive rod with pickup coil and bias magnets

$>$ Terfenol-D $\left(\mathrm{Tb}_{0.3} \mathrm{Dy}_{0.7} \mathrm{Fe}_{1.92}\right)$

- Bias stress and shunt resistance were optimized for maximum damping at $750 \mathrm{~Hz}$.

- Carefully controlled load frame experiments $\rightarrow$ dynamic force applied up to $1000 \mathrm{~Hz}$.

\section{METRICS}

Effective compressive modulus
Total loss factor

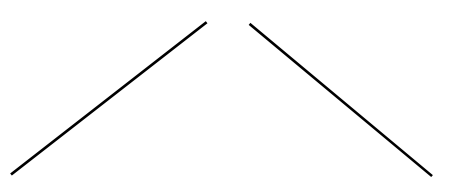

Internal loss factor Shunt loss factor 


\section{Conclusions}

- Unique/accurate data set for validating piezoelectric and magnetostrictive models.

- All devices: Reasonable for driveline damping application

- Moduli 1 order of magnitude lower than steel ( 3 orders higher than rubber)

- Loss factors on the same order as rubber

- Single crystal: Highest shunt loss factor- best tunable damper or energy harvester

- Terfenol-D: Highest total loss factor- best non-tunable damper

- Unintentional eddy current losses due to aluminum magnet holder

- Reconfigure device in 2 ways

1. Non-conductive magnet holder $\rightarrow$ increasing tuning and energy harvesting

2. Get rid of coil and shunt $\rightarrow$ more compact/simpler device.

Would continue to be an effective damper at high frequencies. 
